\title{
PARÂMETROS DE QUALIDADE DO PÃO FRANCÊS
}

\author{
SILA MARY RODRIGUES FERREIRA * \\ PATRÍCIA V. DE OLIVEIRA ** \\ DANIELA PRETTO **
}

Devido à ausência de metodologia para avaliação da qualidade do pão francês em Sistema de Alimentação Coletiva, o presente trabalho teve como objetivo avaliar os parâmetros físicos, físico-químicos e sensoriais do pão francês, comercializado em cinco panificadoras de Curitiba. Nas análises físicas, em cinco repetições, as amostras foram submetidas à determinação do peso, volume, volume específico, densidade e peso seco. As análises físicoquímicas foram realizadas em triplicata de acordo com procedimento da Association of Official Analytical Chemists (AOAC). Para avaliar a qualidade do pão foi utilizada a metodologia do sistema de pontuação global, mediante análise sensorial dos seguintes atributos: cor da crosta, forma e simetria, características da crosta, aspecto da pestana, aspecto de quebra da crosta, cor do miolo, porosidade, textura, aroma e sabor. Atribuiu-se pontuação (de 0 a 5) para cada variável, a qual foi multiplicada pelo fator que expressa a importância relativa de cada variável (totalizando 100 pontos), obtendo-se a classificação dos produtos pela soma dos pontos. Os resultados dos testes físicos e físicoquímicos das cinco amostras de pão francês apresentaram média de $52,23 \mathrm{~g}$ de peso, $31,98 \%$ de umidade, $68,02 \%$ de extrato seco, $35,49 \mathrm{~g}$ de peso seco, $371,8 \mathrm{~cm}^{3}$ de volume, $7,23 \mathrm{~cm}^{3} / \mathrm{g}$ de volume específico e $0,15 \mathrm{~g} / \mathrm{cm}^{3}$ de densidade. As amostras XA, XB e XC apresentaram os melhores resultados podendo ser consideradas como as melhores. $\mathrm{Na}$ classificação pelo sistema de pontuação global, as amostras apresentaram diferença em nível de $1 \%$ de significância em relação à qualidade. As amostras XA e XB apresentaram boa qualidade, seguidas pelas amostras $X C \mathrm{e}$ XE (regular), sendo a amostra XD classificada como ruim. Os resultados obtidos permitem sugerir a adoção desta metodologia de avaliação para seleção de pão francês, em Sistema de Alimentação Coletiva.

\section{PALAVRAS-CHAVE: PÃO FRANCÊS; CONTROLE DA QUALIDADE; ANÁLISE SENSORIAL.}

* Professora de Tecnologia de Alimentos, Departamento de Nutrição, Universidade Federal do Paraná (UFPR). (e-mail: sila@milenio.com.br).

** Estagiárias voluntárias, Laboratório de Tecnologia de Alimentos, Departamento de Nutrição, UFPR. 


\section{INTRODUÇÃO}

Pão francês é o produto fermentado, de forma característica, preparado obrigatoriamente com farinha de trigo, cloreto de sódio e água que apresenta casca crocante, de cor uniforme castanho-dourada, miolo de cor branco-creme, textura e granulação fina não-uniforme (BRASIL, 2000).

A qualidade do pão francês pode ser avaliada mediante análises físicas, físico-químicas, macroscópicas, microscópicas, microbiológicas e sensoriais (BRASIL, 2000b; SÃO PAULO, 1978). As determinações do peso, volume e volume específico devem ocorrer entre duas e 18 horas após o assamento. Para ser considerado de boa qualidade, o pão francês deve apresentar, em média, $50 \mathrm{~g}$ de peso e de 6 a $8 \mathrm{~cm}^{3} / \mathrm{g}$ de volume específico (PIZZINATO et al., [1990?]; BRASIL, 1997). Pesquisando a qualidade nutricional, FISBERG \& COZZOLINO (1996) analisaram 180 unidades de pão francês e encontraram peso médio de $51,09 \mathrm{~g} \pm 2,02 \mathrm{e}$ $28,66 \%$ de umidade.

Volume muito pequeno ou excessivo está ligado à qualidade, quantidade dos ingredientes e ao processamento. Volume muito grande pode ser decorrente de fermentação insuficiente ou excessiva, pouco sal, farinha com baixo teor de glúten, glúten muito forte ou muito fraco, farinha com baixo teor de maltose ou baixa hidratação da massa. O volume excessivo pode ser devido, também, à quantidade demasiada de aditivos melhoradores, ou temperatura muito alta do forno (BRASIL, 1997; BRASIL, 2000a; MOINHO RIO NEGRO, 1997; PERFECTA, [1998?]).

A umidade, segundo a RDC n. 90/00 do INMETRO, não deve ultrapassar a 38\%, considerando a massa livre de recheio e cobertura (BRASIL, 2000b). No setor de panificação observa-se tendência em colocar no mercado produtos com maior teor de umidade, aumentando a maciez e conferindo aspecto mais fresco ao pão. A não-conformidade em relação à umidade não representa risco para a saúde dos consumidores, mas aumenta 0 risco de contaminação por bolores (meio propício à proliferação de microrganismos). No caso do pão francês, esta prática não chega a representar risco sob o ponto de vista sanitário, já que o pão francês é consumido muito rápido apresentando tempo curto de permanência na prateleira. Entretanto, o fato apresenta considerável importância sob o ponto de vista econômico (BRASIL, 2000a).

Os parâmetros físicos, físico-químicos e sensoriais têm sido amplamente utilizados para estudar o comportamento de farinha mista em testes de 
panificação. PIZZINATO et al. (1972) testaram o comportamento da farinha mista com 5 e $10 \%$ de quirera de arroz, com diferentes melhoradores em pães tipo francês, mediante determinação do peso, volume e volume específico. PIZZINATO \& VITTI (1975) avaliaram o comportamento da farinha de raspas, associada às farinhas de trigo e soja com adição de diferentes melhoradores, mediante análises físicas, físico-químicas e pelo sistema de pontuação de alguns atributos sensoriais. LEITÃO et al. (1979) estudaram os aspectos referentes à obtenção da farinha de triticale e sua aplicação em produtos de panificação. Após os testes de panificação, os produtos foram submetidos à avaliação de peso, volume, volume específico e análise sensorial da cor, simetria, textura, sabor e aceitação geral.

GUTKOSKI et al. (1993) investigaram a substituição parcial da farinha de trigo por farinha de aveia em zero, 5, 10, 15 e 20\%, efetuando análises de peso, volume, volume específico e sensorial das amostras. CORRALLES et al. (1993) avaliaram a substituição de bromato de potássio por ácido ascórbico em pão francês pelas propriedades sensoriais e físico-químicas dos produtos obtidos. FREITAS et al. (1997) utilizaram a determinação do peso, volume específico e análise sensorial para avaliar o desempenho da farinha de trigo mista com zero, 10, 20,30 e 40\% de farinha de mandioca na elaboração de pães de fôrma mistos. GRANITO \& GUERRA (1997) usaram o peso, volume, peso específico e a textura instrumental para investigar as propriedades físico-químicas e de qualidade de pães elaborados com farinhas mistas de 10 e $15 \%$ de farinha de gérmen de trigo, utilizando diferentes melhoradores.

$\mathrm{Na}$ análise sensorial são avaliadas as características externas (cor, natureza da crosta, simetria e pestana) e as características internas (textura, cor do miolo e estrutura da célula do miolo), além do aroma e sabor (DUTCOSKI, 1996; ELÍAS \& CONDÉ 1985; MOINHO RIO NEGRO, 1997; GUTKOSKI et al., 1997; BRASIL, 2000).

O pão deve apresentar aparência de massa cozida, simetria, cor, aroma e sabor característicos, além de duas crostas, uma exterior, que ao ser manipulada pode soltar-se e, a outra, mais consistente, bastante aderida ao miolo. A cor da parte externa superior deve ser dourada/amarelada, natural, uniforme e da parte inferior, marrom. Casca sem brilho ou opaca se deve à falta de vapor no forno, formação de casca no pão cru ou falta de açúcares residuais na formulação. A espessura da casca deve medir, aproximadamente, de 01 a 02 mm (MOINHO RIO NEGRO, 1997; PERFECTA, [1998?]; PIZZINATO et al., [1990?]). O miolo deve apresentar cor branco-creme uniforme, ser poroso, leve, homogêneo e elástico, não 
deve aderir aos dedos quando comprimido, nem apresentar aglomerações duras, pontos negros, pardos ou avermelhados. O pão de boa qualidade apresenta miolo consistente, cavidades irregulares, textura macia e aveludada, sedosa e elástica. Miolo frágil ou com buracos pode ser decorrente de erros na formulação e no processamento, tais como: excesso de melhoradores, mistura inadequada, massa muito dura, moldagem malfeita e fermentação excessiva (MOINHO RIO NEGRO, 1997; PERFECTA, [1998?]; PIZZINATO et al., [1990?]).

O pão ruim apresenta miolo muito branco ou malcozido, formado por pequenas cavidades, o que lhe dá aspecto de esponja e massa pegajosa. A cor da crosta é amarelada ou queimada e a textura áspera, dura, esfarelada e empelotada. Pão que não apresente as características de acordo com o padrão de qualidade deve ser rejeitado (MOINHO RIO NEGRO, 1997; BRASIL, 2000a).

Segundo KENT (1987) a cor do miolo é afetada diretamente pelas características da farinha, assim como pela granulosidade da massa. Quanto mais fina a granulosidade, mais brilhante será a cor. A análise deve ser efetuada imediatamente após o corte, pois o miolo tende a escurecer um pouco após exposição prolongada ao ambiente.

A pestana do pão francês cumpre função no desenvolvimento da massa e no aspecto exterior do pão. Cada incisão cria uma área de menor resistência que facilita e normaliza a ação do desenvolvimento gasoso, aumentando as possibilidades de crescimento da massa. Boa pestana deve ser fina e apresentar abertura uniforme. Pestana fechada ou muito grossa indica problemas técnicos na elaboração. Pestana fechada aponta para modelagem e corte malfeitos, excesso de umidade na câmara, fermentação em demasia ou massa fraca. Pestana muito grossa pode resultar de cortes muito profundos na massa, pouco crescimento ou massa muito forte (MOINHO RIO NEGRO, 1997; PERFECTA, [1998?]; PIZZINATO et al., [1990?]).

A forma ou simetria deve ser uniforme e bem-definida, caso contrário indica manuseio e processamento inadequados. Também pode resultar de massa muito dura com baixo teor de água, assim como fermentação inadequada, insuficiente ou manuseio incorreto e grosseiro (KENT, 1987; PIZZINATO et al., [1990?]; MOINHO RIO NEGRO, 1997).

O pão com boas características de qualidade requer desempenho adequado em relação ao desenvolvimento da massa. Este envolve os seguintes 
fatores: concentração de gás, elasticidade e resistência da massa, e, capacidade de retenção de gás. O desenvolvimento e aspecto do pão dependem da qualidade e da quantidade dos ingredientes da massa e do emprego de boa tecnologia (HOSENEY, 1991).

Para avaliar a qualidade do pão vem sendo utilizado o sistema de pontuação global que foi empregado por EL-DASH, (1978) e depois por vários pesquisadores (ELÍAS \& CONDÉ, 1985; BAIÃO et al., 1987; GUTKOSKI et al., 1997). O valor obtido pelos atributos (volume, cor da crosta, quebra, simetria, pestana, estrutura das células do miolo, cor do miolo, textura do miolo, sabor e aroma na análise sensorial) é multiplicado por um fator (totalizando 100 pontos), que depois de somados fornecem a classificação do produto (GUTKOSKI et al., 1997; DUTCOSKI, 1996; ELÍAS \& CONDÉ, 1985). No ramo de panificação, esta metodologia tem sido empregada para avaliar as características do pão servindo como parâmetro para a tomada de decisões corretivas em relação a defeitos apresentados no produto acabado (MOINHO RIO NEGRO, 1997).

ELÍAS \& CONDÉ (1985) aplicaram o sistema de valorização global na avaliação de quatro farinhas com diferentes tempos, fermentações e melhoradores. GUTKOSKI et al. (1997) avaliaram a influência de diferentes melhoradores usando medidas reológicas e teste de panificação. A qualidade dos pães foi definida mediante análise sensorial pela metodologia do sistema de pontuação global dos atributos.

SÁNCHEZ et al. (1998) estudaram o melhoramento da qualidade nutricional do pão tipo francês mediante análises físicas, sensoriais e nutricionais. Para identificar as melhores amostras compararam o volume com a pontuação global.

Segundo FISBERG \& COZZOLINO (1996) o pão francês faz parte da cesta básica do brasileiro. Por ser extremamente consumido pela população infantil, por adolescentes e adultos é considerado alimento universal fazendo parte do desjejum, de lanches ou do acompanhamento das refeições principais. Consiste em fonte de carboidratos e de proteínas com base na principal matéria-prima utilizada. Quando comparado às recomendações diárias para crianças de 4 a 6 anos de vida, dois pães diários na alimentação, segundo os autores, representam 16,8\%, 36,7\%, 25,8\% das necessidades de energia, proteínas e niacina, respectivamente.

O presente trabalho teve como objetivo avaliar os parâmetros físicos, físicoquímicos e sensoriais do pão francês comercializado em cinco panificadoras de Curitiba. 


\section{MATERIAL E MÉTODOS}

\subsection{AMOSTRAGEM}

Foram analisadas amostras de pão francês de 50 gramas, adquiridas em cinco panificadoras de Curitiba, segundo critério de preço (baixo, médio e alto). Os valores das amostras foram: $A=R \$ 0,12 ; B=R \$ 0,10 ; C=$ $R \$ 0,15 ; D=R \$ 0,08$ e $E=R \$ 0,13$.

Na coleta das amostras, nos diferentes locais, seguiu-se o plano de amostragem para tomada de peso, conforme Portaria n. 003/97 do INMETRO. Do estabelecimento que tivesse entre 91 a 150 unidades de pães para a venda no ato da coleta foram retiradas, aleatoriamente, 35 amostras de cada panificadora, no horário entre 8h e 8h30 min.

As amostras foram acondicionadas em pacotes de papel manilhinha e transportadas para o Laboratório de Tecnologia de Alimentos do Departamento de Nutrição. Logo a seguir, foram separadas aleatoriamente para formar subamostras para as análises físicas, físico-químicas e sensoriais. Cinco amostras foram retiradas para as análises físicas, assim como um pão francês, por provador, para a análise sensorial. As subamostras foram acondicionadas nas embalagens de papel manilhinha, identificadas, fechadas e mantidas em local com pouca umidade. As análises das amostras foram conduzidas de forma a não ultrapassar o período de uma ou três horas entre a coleta e as análises.

\subsection{ANÁLISES FÍSICAS E FÍSICO-QUÍMICAS}

Para determinação da umidade e extrato seco as amostras foram trituradas e as alíquotas de cada amostra (em triplicatas), obtidas por quarteamento, submetidas à dessecação em estufa com ventilação a $105^{\circ} \mathrm{C}$ até peso constante, segundo AOAC (2000). O peso seco foi obtido por diferença.

As análises físicas das amostras, conduzidas com cinco repetições, envolveram as determinações de massa, volume, volume específico e densidade. A massa das amostras foi verificada, individualmente, em balança eletrônica digital. O volume foi determinado por deslocamento com sementes tendo como princípio a técnica de deslocamento em água com vazão de fluxo, em equipamento próprio, descrita por ELÍAS \& CONDÉ (1985). Em um recipiente tarado com semente de painço, colocado no interior de outro de maior diâmetro, a amostra foi acomodada e as 
sementes, do recipiente tarado, foram despejadas com auxílio de funil até o transbordamento. Em seguida, o recipiente foi nivelado com auxílio de régua e o volume transbordado (volume da amostra) foi medido em proveta de $100 \mathrm{~mL}$. O volume específico foi determinado segundo o método descrito por GRISWOLD (1972) e LEITÃO (1979), mediante relação volume/ massa da amostra. A densidade foi determinada pela relação massa/ volume.

\subsection{ANÁLISE SENSORIAL}

A análise sensorial foi realizada no Laboratório de Análise Sensorial do Departamento de Nutrição, por equipe de 12 provadores treinados. Cada julgador avaliou todas as amostras, devidamente codificadas, em sessões diferentes. Empregou-se a análise descritiva quantitativa (ADQ), segundo a ABNT (1998), com escala estruturada de cinco pontos (Quadro 1). Os provadores foram selecionados em função da experiência, conhecimento técnico e habilidade em discriminar e julgar os atributos.

O instrumento para avaliação sensorial (Quadro 1) foi construído em sessões anteriores aos testes, juntamente com os provadores, levandose em consideração os atributos sensoriais do produto, bem como as características relevantes observadas pelos provadores. Durante as sessões de treinamento foram definidos os atributos, seus significados, a seqüência e o valor atribuído aos mesmos.

Nas sessões de elaboração do instrumento, amostras de referência foram apresentadas à equipe para dar noções qualitativa e quantitativa dos atributos avaliados. Para quantificar cada atributo sensorial foi utilizada escala de um a cinco, com a descrição em todos os pontos, com exceção do atributo aspecto de quebra da crosta, cujos valores foram estipulados somente nos limites mínimo e máximo.

Os testes foram realizados em cabines fechadas, com iluminação branca natural. Os provadores receberam amostra do produto em pratinho branco, com faca apropriada para cortá-lo, água, ficha do teste ADQ e o glossário com a escala (Quadro 1). Após o preenchimento dos formulários pelos provadores foi realizada análise estatística dos resultados usando-se a ANOVA (GOMES, 1973). As médias que apresentaram diferença significativa ao nível de $1 \%$ foram identificadas pelo teste de Tukey (MONTEIRO, 1984). 


\section{QUADRO 1 - ATRIBUTOS DA ANÁLISE DESCRITIVA QUANTITATIVA E SUAS RESPECTIVAS PONTUAÇÕES}

\begin{tabular}{|c|c|c|c|c|c|}
\hline Escores & 5 & 4 & 3 & 2 & 1 \\
\hline $\begin{array}{l}\text { Volume } \\
\text { espec fico }\end{array}$ & Muito bom* & Bom* & Regular* $^{*}$ & Muito grande & Muito pequeno \\
\hline Cor da crosta & $\begin{array}{l}\text { Dourada, } \\
\text { natural, } \\
\text { uniforme }\end{array}$ & $\begin{array}{l}\text { Natural, } \\
\text { levemente } \\
\text { tostada }\end{array}$ & $\begin{array}{l}\text { Ligeiramente } \\
\text { alterada (clara } \\
\text { ou escura), } \\
\text { desuniforme }\end{array}$ & $\begin{array}{l}\text { Com algumas } \\
\text { manchas } \\
\text { escuras }\end{array}$ & 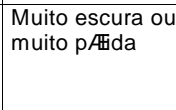 \\
\hline $\begin{array}{l}\text { Forma e } \\
\text { simetria }\end{array}$ & Sim Ørica & $\begin{array}{l}\text { Sim Ørica, } \\
\text { levemente } \\
\text { modificada }\end{array}$ & $\begin{array}{l}\text { Achatada, } \\
\text { assim Ørica }\end{array}$ & 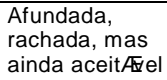 & $\begin{array}{l}\text { Muito } \\
\text { deformada }\end{array}$ \\
\hline $\begin{array}{l}\text { Caracter sticas } \\
\text { da crosta }\end{array}$ & $\begin{array}{l}\text { Fina, macia e } \\
\text { crocante }^{*}\end{array}$ & $\begin{array}{l}\text { Macia e } \\
\text { crocante* }\end{array}$ & $\begin{array}{l}\text { Medianamente } \\
\text { dura* }^{*}\end{array}$ & $\begin{array}{l}\text { Dura ou muito } \\
\text { macia* }^{*}\end{array}$ & $\begin{array}{l}\text { Muito dura ou } \\
\text { borrachenta* }\end{array}$ \\
\hline $\begin{array}{l}\text { Aspecto da } \\
\text { pestana }\end{array}$ & $\begin{array}{l}\text { Aberta* } \\
\text { proeminente } \\
\text { (pestana } \\
\text { levantada), } \\
\text { centralizada }\end{array}$ & Semi-aberta & $\begin{array}{l}\text { Aberta, sem } \\
\text { pestana }\end{array}$ & Fechada* $^{*}$ & Sem corte \\
\hline $\begin{array}{l}\text { Aspecto de } \\
\text { quebra da } \\
\text { crosta }\end{array}$ & Uniforme ${ }^{*}$ & & & & Desuniforme $^{\star}$ \\
\hline Cor do miolo & $\begin{array}{l}\text { Uniforme, } \\
\text { branco ou } \\
\text { levemente } \\
\text { creme }\end{array}$ & $\begin{array}{l}\text { Uniforme, } \\
\text { creme }\end{array}$ & $\begin{array}{l}\text { Uniforme, } \\
\text { ligeiramente } \\
\text { escurecida }\end{array}$ & $\begin{array}{l}\text { Desuniforme } \\
\text { com algumas } \\
\text { manchas }\end{array}$ & Escuro* \\
\hline Porosidade & \begin{tabular}{|l|} 
CØulas \\
ovaladas, \\
uniformes, sem \\
buracos
\end{tabular} & $\begin{array}{l}\text { CØulas } \\
\text { ovaladas, } \\
\text { abertas } \\
\text { uniformes, com } \\
\text { pequenos } \\
\text { buracos } \\
\end{array}$ & $\begin{array}{l}\text { C@ulas } \\
\text { uniformes, } \\
\text { pouco } \\
\text { fechadas }\end{array}$ & $\begin{array}{l}\text { CØulas muito } \\
\text { fechadas, } \\
\text { paredes } \\
\text { grossas }\end{array}$ & $\begin{array}{l}\text { CØulas muito } \\
\text { abertas, } \\
\text { paredes finas e } \\
\text { buracos }\end{array}$ \\
\hline Textura & \begin{tabular}{|l} 
Sedosa, \\
granula a o \\
uniforme
\end{tabular} & $\begin{array}{l}\text { Ligeiramente } \\
\text { desuniforme }\end{array}$ & $\begin{array}{l}\text { Desuniforme e } \\
\text { Aspera }\end{array}$ & $\begin{array}{l}\text { Desuniforme, } \\
\text { pegajosa ou } \\
\text { seca }\end{array}$ & $\begin{array}{l}\text { Muito dura ou } \\
\text { muito seca }\end{array}$ \\
\hline Aroma & $\begin{array}{l}\text { Espec fico, } \\
\text { mas agrad/Fel }\end{array}$ & Espec fico bom & $\begin{array}{l}\text { Ligeiramente } \\
\text { alterado, } \\
\text { aceit/的l }\end{array}$ & $\begin{array}{l}\text { Alterado, } \\
\text { ran oso ou } \\
\text { com aroma de } \\
\text { fermento } \\
\end{array}$ & $\begin{array}{l}\text { Alterado, } \\
\text { at pico, Ateido }\end{array}$ \\
\hline Sabor & $\begin{array}{l}\text { Espec fico, } \\
\text { excepcional- } \\
\text { mente } \\
\text { agrad/Æel }\end{array}$ & Espec fico & $\begin{array}{l}\text { Levemente } \\
\text { alterado, } \\
\text { aceit/बel }\end{array}$ & $\begin{array}{l}\text { Alterado, } \\
\text { levemente } \\
\text { ins pido ou } \\
\text { amargo } \\
\end{array}$ & $\begin{array}{l}\text { Completamente } \\
\text { alterado, azedo } \\
\text { ou outro sabor } \\
\text { estranho }\end{array}$ \\
\hline
\end{tabular}

Para construção do instrumento foram seguidas as pontuações (escala de $0-5$ ) sugeridas pelo MOINHO RIO NEGRO (1997) e as descrições definidas nas sessões anteriores com os provadores.

Os asteriscos $\left({ }^{*}\right)$ indicam as descrições utilizadas pelo MOINHO RIO NEGRO (1997). 
A classificação do volume específico (Tabela 1) resultou da combinação da proposta do MOINHO RIO NEGRO (1997) com a análise dos provadores em relação à expectativa do volume do pão francês. Cada volume específico correspondeu a um atributo com valor respectivo.

\section{TABELA 1 - CLASSIFICAÇÃO DO VOLUME ESPECÍFICO DO PÃO FRANCÊS}

\begin{tabular}{c|l}
\hline VOLUME ESPEC"FICO & CLASSIFICA ^ $^{\star}$ O \\
\hline Entre 6 e 8 & Muito bom (5 pontos) $^{\star}$ \\
Entre 5 e 6 & Bom (4 pontos) $^{\star}$ \\
Entre 4 e 5 & Regular (3 pontos) \\
Acima de 8 & Muito grande (2 pontos) \\
Abaixo de 4 & Muito pequeno (1 ponto) ${ }^{\star}$ \\
\hline
\end{tabular}

(*) Classificação e pontuação conforme MOINHO RIO NEGRO (1997).

Para avaliar a qualidade das amostras foram relacionadas as variáveis de qualidade versus fator (Quadro 2). O fator correspondente é resultante da análise contextualizada dos atributos avaliados pelos provadores em combinação com os fatores de qualidade atribuídos por DUTCOSKY, (1996); ELÍAS \& CONDÉ (1985). A pontuação de 0 a 5, definida para cada qualidade, foi multiplicada pelo fator que aparece na coluna da direita do Quadro 2 que expressa a importância relativa de cada qualidade.

\section{QUADRO 2 - FATOR DAS VARIÁVEIS DE QUALIDADE DO PÃO FRANCÊS}

\begin{tabular}{|l|l|}
\hline QUALIDADE & FATOR \\
\hline Volume & $(x 3)$ \\
Corda crosta & $(x 2)$ \\
Forma e simetria & $(x 2)$ \\
Caracter sticas da crosta & $(x 2)$ \\
Aspectoda pestana & $(x 1)$ \\
Aspecto de quebra & $(x 1)$ \\
Cordo miolo & $(x 1)^{a}$ \\
Porosidade & $(x 2)^{a, b}$ \\
Textura & $(x 2)$ \\
A roma & $(x 2)^{a}$ \\
Sabor & $(x 2)$ \\
\hline
\end{tabular}

a Retirado de DUTCOSKY, (1996); ' Subtraído de ELÍAS \& CONDÉ (1985). 


\subsection{CLASSIFICAÇÃO DAS AMOSTRAS}

A soma de todos os pontos obtidos fornece a pontuação global máxima de 100 pontos para o produto. O valor da pontuação máxima permite classificar o pão francês, conforme mostra a Tabela 2.

\section{TABELA 2 - CLASSIFICAÇÃO DAS AMOSTRAS}

\begin{tabular}{|c|c|}
\hline $\mathrm{PONTUA}^{\wedge}{ }^{\wedge} \mathrm{O}$ & $\mathrm{CLASSIFICA}^{*} \mathrm{O}$ \\
\hline 81 a 100 & $\mathrm{P}^{\mathrm{a}} \mathrm{o}$ de boa qualidade \\
\hline 61 a 80 & Regular \\
\hline 31 a 60 & Ruim \\
\hline Menos de 30 & Qualidade inaceit/『/el \\
\hline
\end{tabular}

FONTE: DUTCOSKY, 1996.

\section{RESULTADOS E DISCUSSÕES}

Os resultados das análises físicas e físico-químicas das amostras coletadas em cinco panificadoras de Curitiba estão apresentados na Tabela 3.

\section{TABELA 3 - ANÁLISES FÍSICAS E FÍSICO-QUÍMICAS DO PÃO FRANCÊS}

\begin{tabular}{l|lllllll}
\hline Caracter sticas & ${ }^{*}$ X A & ${ }^{*}$ X B & ${ }^{*}$ X C & ${ }^{*}$ X D & ${ }^{*}$ X E & MØdia \\
\hline Peso (g) & 53,24 & 51,31 & 51,86 & 57,09 & 47,65 & 52,23 \\
Umidade (\%) & 31,5 & 33,5 & 30,5 & 36,0 & 28,4 & 31,98 \\
Extrato Seco (\%) & 68,5 & 66,5 & 69,5 & 64,0 & 71,6 & 68,02 \\
Peso seco (g) & 36,47 & 34,12 & 36,21 & 36,54 & 34,12 & 35,49 \\
Volume $\left(\mathrm{cm}^{3}\right)$ & 332 & 391 & 326 & 303 & 507 & 371,8 \\
Volume espec fico & 6,24 & 7,64 & 6,29 & 5,31 & 10,65 & 7,23 \\
$\left(\mathrm{~cm}^{3} / \mathrm{g}\right)$ & 0,16 & 0,13 & 0,16 & 0,19 & 0,09 & 0,15 \\
Densidade $\left(\mathrm{g} / \mathrm{cm}^{3}\right)$ & 0,16 & 0,10 & 0,15 & 0,08 & 0,13 & 0,12 \\
Pre o unit/Æio $(\mathrm{R} \$)$ & 0,12 & & & & &
\end{tabular}

${ }^{*} \mathrm{X}=$ Média das amostras de cada panificadora. 
Conforme pode ser observado na Tabela 3, o peso da maioria das amostras aproximou-se do peso mínimo (50 g) estabelecido pela Portaria n. 003/97 do INMETRO. As médias das amostras XB e XC mostraram-se similares ao peso médio de 51,09 gramas, encontrado por FISBERG \& COZZOLINO (1996). A amostra XD superou em $14,0 \%$ a quantidade exigida pela legislação vigente, ao contrário da amostra XE que apresentou déficit de $4,7 \%$ em relação ao peso.

Todas as amostras apresentaram valores enquadrados no limite de até $38,0 \%$ de umidade, definido pela Portaria n. 90/00 (BRASIL, 2000b). Os valores encontrados mostraram-se acima do resultado de $28,66 \%$ reportados por FISBERG \& COZZOLINO (1996) em 180 unidades de pão francês.

As amostras de pão francês apresentaram teor de 64,0 a $71,6 \%$ de extrato seco, com variação de 34,12 a $36,54 \mathrm{~g}$, do peso seco (Tabela 1). Os valores encontrados evidenciaram similaridade entre as amostras, independente do peso inicial, mesmo para as amostras XD e XE (pesos extremos). A amostra XD apresentou o maior teor de umidade $(36,0 \%) \mathrm{e}$ a XE o menor $(28,4 \%)$.

Considerando que o pão francês deve conter 50 gramas de peso e no máximo 38,0\% de umidade (Portaria INMETRO n. 003/97 e RDC n. 90/00, respectivamente), uma unidade de pão deve apresentar peso seco de, no mínimo, 31,0\%. A média dos resultados das amostras analisadas evidenciou valores acima deste limite.

O volume das amostras variou de 303 a $507 \mathrm{~cm}^{3}$. SÁNCHEZ et al. (1998), estudando o melhoramento da qualidade nutricional de pão francês com adição de outras farinhas, encontraram volume de $377 \mathrm{~cm}^{3}$. A amostra XD apresentou maior peso $(57,09 \mathrm{~g})$ e menor volume $\left(303 \mathrm{~cm}^{3}\right)$, enquanto que a amostra XE de menor peso $(47,65 \mathrm{~g})$ apresentou maior volume $\left(507 \mathrm{~cm}^{3}\right)$. O volume está relacionado com a qualidade e quantidade dos ingredientes, principalmente os melhoradores e também com o processamento do produto (MOINHO RIO NEGRO, 1997; GÓMEZ et al., 1998; GROSSMANN \& BARBER, 1997).

O volume específico encontrado nas amostras XA, XC e XD (5,31 a $6,29 \mathrm{~cm}^{3} / \mathrm{g}$ ) mostrou-se próximo aos limites de 4,61 a $5,83 \mathrm{~cm}^{3} / \mathrm{g}$ verificados por LEITÃO et al. (1979), de 4,87 a $5,81 \mathrm{~cm}^{3} / \mathrm{g}$, relatados por GUTKOSKI et al. (1993) e acima dos valores de 2,25 a $3,25 \mathrm{~cm}^{3} / \mathrm{g}$ reportados por FREITAS et al. (1997). GÓMEZ et al. (1998) encontraram 
volume específico de $5,74 \mathrm{~cm}^{3} / \mathrm{g}$. As amostras XB e XE apresentaram 7,64 e $10,65 \mathrm{~cm}^{3} / \mathrm{g}$ de volume específico, respectivamente, superando todos os valores encontrados na literatura citada. Segundo EL DASH (1983), citado por GUTKOSKI et al. (1993), o pão adequado deve apresentar volume específico acima de $6,0 \mathrm{~cm}^{3} / \mathrm{g}$. Para o MOINHO RIO NEGRO (1997) a faixa ideal encontra-se entre 4 e $8 \mathrm{~cm}^{3} / \mathrm{g}$, na qual se enquadraram as amostras XA, XB, XC e XD (Tabela 4). O volume é de grande importância na determinação da qualidade do pão francês, pois resulta da qualidade dos ingredientes usados na formulação da massa, em especial a farinha, assim como melhoradores e tratamentos empregados durante a elaboração. Pão com volume excessivamente grande apresenta textura fraca, com granulosidade grosseira, não sendo aceitável como produto de boa qualidade (MOINHO RIO NEGRO, 1997; GUTKOSKI et al., 1997).

A densidade das amostras (variação entre 0,09 a $0,19 \mathrm{~g} / \mathrm{cm}^{3}$ ) aproximouse do valor $\left(0,17 \mathrm{~g} / \mathrm{cm}^{3}\right)$ encontrado por GÓMEZ et al. (1998).

O preço das amostras variou de $R \$ 0,08$ a $R \$ 0,15$, com média de $R \$ 0,12$. As melhores amostras, $X A, X B$ e $X C$, custaram entre $R \$ 0,10$ e $R \$ 0,15$. As amostras $\mathrm{XD}$ e XE, consideradas piores, em relação aos resultados físicos e físico-químicos, custaram $R \$ 0,08$ e $R \$ 0,13$, respectivamente.

\section{TABELA 4 - CLASSIFICAÇÃO DO VOLUME ESPECÍFICO DAS AMOSTRAS}

\begin{tabular}{c|c|c}
\hline AMOSTRA & VOLUME ESPEC"FICO $\left(\mathrm{cm}^{3} / \mathrm{g}\right)$ & CLASSIFICA`^O \\
\hline XA & 6,24 & Muito bom \\
XB & 7,64 & Muito bom \\
XC & 6,29 & Muito bom \\
XD & 5,31 & Bom \\
XE & 10,65 & Muito grande \\
\hline
\end{tabular}

A classificação das amostras na análise sensorial (Tabela 5) foi obtida pela soma dos valores dos atributos, após multiplicação pelo fator de qualidade correspondente (Quadro 2).

As amostras $X A$ e $X B$ destacaram-se em relação à qualidade, porém são estatisticamente iguais às amostras XC e XE, diferindo da amostra XD. Já as amostras XC, XD e XE não apresentaram diferenças estatisticamente significativas entre si $(p \leq 0,01)$. 


\section{TABELA 5 - CLASSIFICAÇÃO DAS AMOSTRAS NA ANÁLISE SENSORIAL}

\begin{tabular}{c|c|c}
\hline AMOSTRAS & PONTUA $^{*} \mathrm{O}^{\circ}$ & CLASSIFICA ^^ $^{*}$ \\
\hline $\mathrm{XA}_{\mathrm{y}}$ & 81,8 & $\mathrm{P}^{\mathrm{a}}$ o de boa qualidade \\
$\mathrm{XB}_{\mathrm{y}}$ & 82,8 & $\mathrm{P}^{\mathrm{a}}$ o de boa qualidade \\
$\mathrm{XC}_{\mathrm{y}, \mathrm{z}}$ & 74,8 & Regular \\
$\mathrm{XD}_{z}$ & 59,0 & Ruim \\
$\mathrm{XE}_{\mathrm{y}, \mathrm{z}}$ & 67,0 & Regular \\
\hline
\end{tabular}

Amostras seguidas por letras iguais não diferem entre si pelo teste de Tukey $(p \leq 0,01)$.

As médias encontradas para o pão francês de 50 gramas enquadraramse nos limites de 53,0 a 86,0 pontos, encontrados por ELÍAS \& CONDÉ (1985) para pão francês tipo bengala de, aproximadamente, $300 \mathrm{e}$ 350 gramas. GUTKOSKI et al. (1997) utilizaram o sistema de pontuação global para verificar a influência de melhoradores em farinhas mistas e encontraram valores de 68,52 a 74,91 pontos nas diferentes formulações.

SÁNCHEZ et al. (1998) encontraram pontuação de 92,0 e volume de $377 \mathrm{~cm}^{3}$ para o pão francês elaborado com farinha de trigo. A qualidade e quantidade dos ingredientes da formulação, como a forma de processamento, podem produzir diminuição no volume e perda das características externas e internas do pão, de tal forma que reduzem a pontuação global (MOINHO RIO NEGRO, 1997; SÁNCHEZ et al.,1998; GROSSMANN \& BARBER, 1997).

Apenas as amostras XD e XE mostraram-se diferentes das demais e dos dados fornecidos pela literatura. A amostra XE pode ser considerada de baixa qualidade, pois apresentou o menor peso apesar do seu maior volume específico. A amostra XD com maior peso e volume específico mais baixo também mostrou qualidade inferior. Em relação ao custo, a amostra XD com menor preço $(R \$ 0,08)$ sugere utilização de ingredientes de baixa qualidade na formulação, quantidade inadequada e/ou processamento incorreto. Os resultados obtidos para a amostra XE, com preço unitário de $R \$ 0,12$, revelaram que nem sempre o preço é indicativo de qualidade. 


\section{CONCLUSÃO}

As amostras $\mathrm{XA}, \mathrm{XB}$ e $\mathrm{XC}$ apresentaram resultados superiores em qualidade nas análises físicas, podendo ser consideradas como as melhores.

Na classificação pelo sistema de pontuação global, as amostras XA e XB evidenciaram boa qualidade seguidas pelas amostras XC e XE com qualidade regular, sendo a amostra XD classificada como ruim.

Os preços mais baixos corresponderam às amostras XD e XB.

Tendo em vista a ausência de parâmetros físicos para seleção do pão francês, em Sistema de Alimentação Coletiva, pode-se dizer que o pão francês de boa qualidade deve apresentar $50 \mathrm{~g}$ de peso, 300 a $400 \mathrm{~cm}^{3}$ de volume, 0,13 a $0,16 \mathrm{~g} / \mathrm{cm}^{3}$ de densidade, de 4 a $8 \mathrm{~cm}^{3} / \mathrm{g}$ de volume específico e até $38 \%$ de umidade. Na classificação pelo sistema de pontuação global deve atingir de 81 a 100 pontos.

Em razão dos resultados encontrados neste trabalho sugere-se que esta metodologia seja empregada na seleção de pão francês em Sistema de Alimentação Coletiva.

\section{Abstract}

\section{QUALITY PATTERNS OF FRENCH BREAD}

Due to the absence of a methodology to evaluate the quality of French bread in a Production of Collective Meals System, the present work had as objective to evaluate the quality, physical, physical-chemical and sensorial parameters of the French bread marketed in five bakeries in Curitiba (Brazil). In the physical analyses, with five repetitions, the samples were submitted to the determination of weight, volume, specific volume, density and dry weight. The physical-chemical analysis was accomplished with three replicates in agreement with the AOAC procedure. To evaluate the quality of the bread, the methodology of global punctuation system was used, by sensorial analysis of the following attributes: color of the crust, form and symmetry, characteristics of the crust, aspect of the lash, aspect of crust break, color of the crumb, porosity, texture, aroma and flavor. For each variable a grade (from 0 to 5) was attributed, which was multiplied by a factor that expressed the relative importance of each variable (to a total of 100 points), obtaining the product classification by the sum of the points. The results of the physical and physical-chemical tests of the five samples of French bread presented an average of $52,23 \mathrm{~g}$ of weight; $31,98 \%$ of humidity, $68,02 \%$ of dry extract, $35,49 \mathrm{~g}$ of dry weight, $371,8 \mathrm{~cm}^{3}$ of volume, $7,23 \mathrm{~cm}^{3} / \mathrm{g}$ of specific volume and $0,15 \mathrm{~g} / \mathrm{cm}^{3}$ of density. The samples $\mathrm{XA}, \mathrm{XB}$ and $\mathrm{XC}$ presented better results and could be considered as the best. In the classification using the global punctuation system, the samples presented difference 
at the level of $1 \%$ of significance in relation to the quality. The samples $X A$ and $X B$ presented good quality, followed by the samples XC and XE (regular), being the sample $X D$ classified as bad. The results obtained allow suggest the evaluation methodology for selection of French bread in a Production of Collective Meals System.

KEY-WORDS: FRENCH BREAD; QUALITY CONTROL; SENSORIAL ANALYSIS.

\section{REFERÊNCIAS}

1 ASSOCIAÇÃO BRASILEIRA DE NORMAS TÉCNICAS. NBR 14140: alimentos e bebidas: análise sensorial: teste de análise descritiva quantitativa (ADQ). Rio de Janeiro, 1998.

2 ASSOCIATION OF OFFICIAL ANALYTICAL CHEMISTS. Official methods of analysis of AOAC international. $17^{\text {th }}$ ed. Gaythersburg, 2000.

$3 \quad$ BAIÃO, V.B.; GOMES, J.C.; MARTYN, M.E.L.; MAFFIA, L.M. Avaliação nutricional de pão francês da farinha composta de trigoalgarroba. Arquivos Biológicos de Tecnologia, Curitiba, v. 30, n. 2, p. 327-336, jun. 1987.

4 BRASIL. Instituto Nacional de Metrologia, Normalização e Qualidade Industrial. Portaria n. 003 de 10 de janeiro de 1997. Dispõe sobre a comercialização do pão francês, ou de sal, a peso (pesagem na presença do consumidor) ou unidades de peso nominal definido. Diário Oficial [da] República Federativa do Brasil, Brasília, 15 jan. 1997. Seção 1, p. 816.

5 BRASIL. Instituto Nacional de Metrologia, Normalização e Qualidade Industrial. Ensaios realizados e resultados observados para o pão de fôrma ou pão para sanduíche. Disponível em:<http:// www.inmetro.gov.br/paoforma.htm>. Acesso em: 25 de maio de 2000a.

6 BRASIL. Ministério da Saúde. Agência Nacional de Vigilância Sanitária. Resolução RDC n. 90, de 18 de outubro de 2000. Aprova o regulamento técnico para fixação de identidade e qualidade de pão. Diário Oficial [da] República Federativa do Brasil, Brasília, out. 2000b. (Também disponível em: <http//www.anvisa.gov.br>. Acesso em: 30 de novembro de 2000).

7 CORRALES, X.; GUERRA, M.; GRANITO, M.; FERRIS, J. Substitución de bromato de potasio por ácido ascórbico en la 
elaboración de pan francés. Archivos Latinoamericanos de Nutricion, Venezuela, v. 43, n. 3, p. 234-240, 1993.

8 DUTCOSKY, S. D. Análise sensorial de alimentos. Curitiba: Champagnat, 1996.

9 EL-DASH, A A. Standardized mixing and fermentation procedure for experimental baking test. Cereal Chemistry, Saint Paul, v. 55, n.4, p. 436-446, 1978.

10 ELÍAS, J. R.; CONDÉ, A. P. El processo de panificacion: etapas fundamentales y papel de la farina en las mismas. Alimentária, v. 22, n.1, p. 17-32, 1985.

11 FISBERG, M.; COZZOLINO, S.M. Valor nutritivo do pão francês. São Paulo: V. Macêdo Alimentos, 1996. (Pesquisa 001).

12 FREITAS, R.E.; STERTZ, S.C.; WASZCZYNSKYJ, N. Viabilidade da produção de pão, utilizando farinha mista de trigo e mandioca em diferentes proporções. Boletim do CEPPA, Curitiba, v. 15, n. 2, p. 197-208, jul./dez.1997.

13 GOMES, F. P. Curso de estatística experimental. 5. ed. Piracicaba: Nobel, 1973. 468 p.

14 GÓMEZ, J.C.; CASTELLANOS, M. R.; SALAZAR, Z.A. Evaluación de las características reológicas y sensoriais de panes elaborados a base de una mezcla de concentrado proteico de girasol y proteína texturizada de soya. Archivos Latinoamericanos de Nutricion, Venezuela, v. 48, n. 2, p.165-168, 1998.

15 GRANITO, M.; GUERRA, M. Efecto del uso de diferentes aditivos de panificación en la calid de panes elaborados com harinas compuestas a base de harina de trigo y germen desgrasado de maíz. Boletim da Sociedade Brasileira de Ciência e Tecnologia de Alimentos, Campinas, v. 17, n. 2, p. 181-187, maio/ago. 1997.

16 GRISWOLD, R.M. Estudo experimental dos alimentos. Rio de Janeiro: Programa de Publicações Didáticas - USAID, 1972.

17 GROSSMANN, M. V. E.; BARBER, C. B. Envejecimento del pan: efecto combinado de $\alpha$-amilasa bacteriana y emulsificante en la textura y en las características amilográficas de la miga. Archivos 
Latinoamericanos de Nutricion, Venezuela, v. 47, n. 3, p.229233, 1997.

18 GUTKOSKI, L.C.; PAVANELLI, A.P.; MIRANDA, M.Z.; CHANG, Y.K. Efeito de melhoradores nas propriedades reológicas e de panificação da massa de farinha de trigo. Boletim da Sociedade Brasileira de Ciência e Tecnologia de Alimentos, Campinas, v. 17, n. 1, p. 11-16, jan./abr. 1997.

19 GUTKOSKI, L.C.; VELLOSO, C.B.; DÓRO, C.T.; SILVEIRA, A.A.E.; BONAPÉ, L. Z. Uso de farinha mista de trigo e aveia em produtos de panificação. Boletim do CEPPA, Curitiba, v. 2, n. 1, p. 33-45, jan./jun. 1993.

20 HOSENEY, R. Carl. Principios de ciencia y tecnologia de los cereales. Zaragoza (España): Acribia, 1991.

21 KENT, N.L. Tecnologia de los cereales. Zaragoza (España): Acribia, 1987.

22 LEITÃO, R. F. de F.; VITTI, P. PIZZINATO, A.; CAMPOS, S.D.S.; MORI, E.E.M.; SIROSE, I.I. Farinha de triticale em panificação. Coletânea do Instituto de Tecnologia de Alimentos, Campinas, v. 10 , p. $45-58,1979$.

23 MOINHO RIO NEGRO. Apostila de panificação. Curitiba, 1997.

24 MONTEIRO, C.L.B. Técnicas de avaliação sensorial. 2. ed. Curitiba: Centro de Pesquisa e Processamento de Alimentos, 1984. $101 \mathrm{p}$.

25 PERFECTA CURITIBA. Noções básicas de panificação. Curitiba, [1998?].

26 PIZZINATTO, A.; LEITÃO, R.F.F.; VITTI, P. Curso de panificação. São Paulo: Secretaria de Agricultura e Abastecimento/Instituto de Tecnologia de Alimentos, [1990?]. $50 \mathrm{p}$.

27 PIZZINATTO, A.; VITTI, P. Pães mistos de trigo, soja e mandioca. Coletânea do Instituto de Tecnologia de Alimentos, Campinas, v. 6, p. 189-203, 1975.

28 PIZZINATTO, A.; VITTI, P.; LEITÃO, R.F.F. Farinha de quirera de arroz em pão francês. Boletim do Instituto de Tecnologia de Alimentos, Campinas, v. 52, p. 131-154, jul./ago. 1972. 
29 SÁNCHEZ. H.D.; OSELLA, C.A.; TORRE, M.A.G. Mejoramiento de la calid nutricional de pan tipo francês. Archivos Latinoamericanos de Nutricion, Venezuela, v. 48, n. 4, p.349353, 1998.

30 SÃO PAULO (Estado). Decreto n. 12.486, de 20 de outubro de 1978. Aprova normas técnicas relativas a alimentos e bebidas. Diário Oficial do Município de São Paulo, São Paulo, p. 251-253, out. 1978. 